\title{
Fluorescein-guided confocal laser endomicroscopy for the detection of ipilimumab-induced colitis
}

Ipilimumab is an antitumor human antibody that has been very recently approved for the treatment of unresectable or metastatic melanoma [1]. By blocking the cytotoxic T-lymphocyte-associated antigen 4 (CTLA4), ipilimumab induces Tcell activation and proliferation, resulting in an enhanced antitumor immune response [2]. The most frequent side effect of this novel therapeutic approach is the development of a T-cell-mediated colitis, marked by severe diarrhea (over $30 \%$ of patients) [3]. Ipilimumab-induced iatrogenic colitis resembles the mucosal inflammatory reaction in the acute phase of inflammatory bowel disease and can even lead to spontaneous intestinal per-

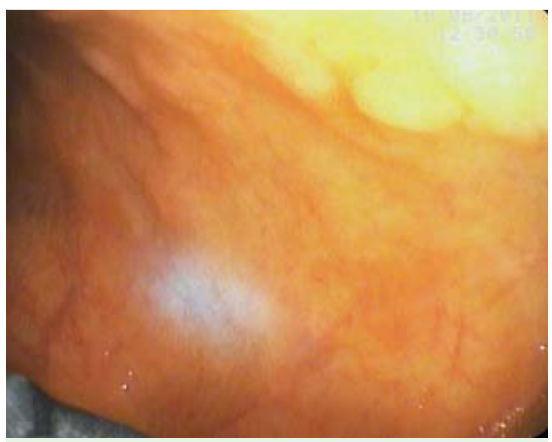

Fig. 1 White light colonoscopy of the sigmoid colon showing faint mucosal edema and erythema in a 71-year-old man with metastatic melanoma, who developed diarrhea after initiation of ipilimumab treatment. foration [4]. Therefore, it is mandatory to have a minimally invasive, evidencebased diagnostic modality for use in the early stages of treatment, which would allow immediate diagnosis and therefore the prompt initiation of necessary immunosuppressive treatment (steroids or antitumor necrosis factor (TNF) antibodies). In this regard, confocal laser endomicroscopy (CLE), a novel endoscopic imaging technique, could be particularly valuable, as it permits real-time visualization of cellular details of the mucosa comparable with conventional histological examination [5]. Several entities of colonic inflammation have been described endopathologically, but so far the value of CLE in describing this novel ipilimumab-induced colitis has not been addressed.

Here, we present the case of a 71-year-old male patient with metastatic melanoma who developed severe watery diarrhea after initiation of ipilimumab treatment. As repeated microbiological stool analysis was negative and ipilimumab-induced colitis was considered possible, the patient was referred to our endoscopy unit. Conventional white light endoscopy showed erythema and mucosal edema mainly in the sigmoid colon ( $\bullet$ Fig. 1 ). Subsequently, we performed fluorescein-guided CLE of the sigmoid mucosa, highlighting inflammatory signs such as altered crypt architecture ( $\bullet$ Fig.2a), inflammatory in- filtrates ( $\bullet$ Fig. 2 b), and fluorescein leakage in the lamina propria ( Fig.2c). With the help of conventional biopsies from the areas analyzed by confocal imaging, we were able to correlate and verify the endomicroscopic findings with conventional histopathology, showing a focal and superficial hemorrhagic and edematous inflammation of the mucosa

\section{(๑ Fig.3).}

The inflammatory features described here highlight the clinical impact of using CLE for diagnosing ipilimumab-induced colitis in vivo, in real time by a non-invasive method. In this particular setting (ipilimumab therapy followed by onset of acute diarrhea and negative stool analysis) endomicroscopy of the colon might be considered as a diagnostic tool for providing endopathological evidence of mucosal inflammation. Endomicroscopy may also provide important diagnostic and outcome benefits to these patients (who already have a high risk of perforation), as by avoiding or reducing the need to take mucosal biopsies, it helps avert potential biopsy-related complications which might require surgical management and prolonged hospitalization (e.g. bleeding or perforation).

\section{Endoscopy_UCTN_Code_CCL_1AD_2AJ}

\section{Competing interests: None}
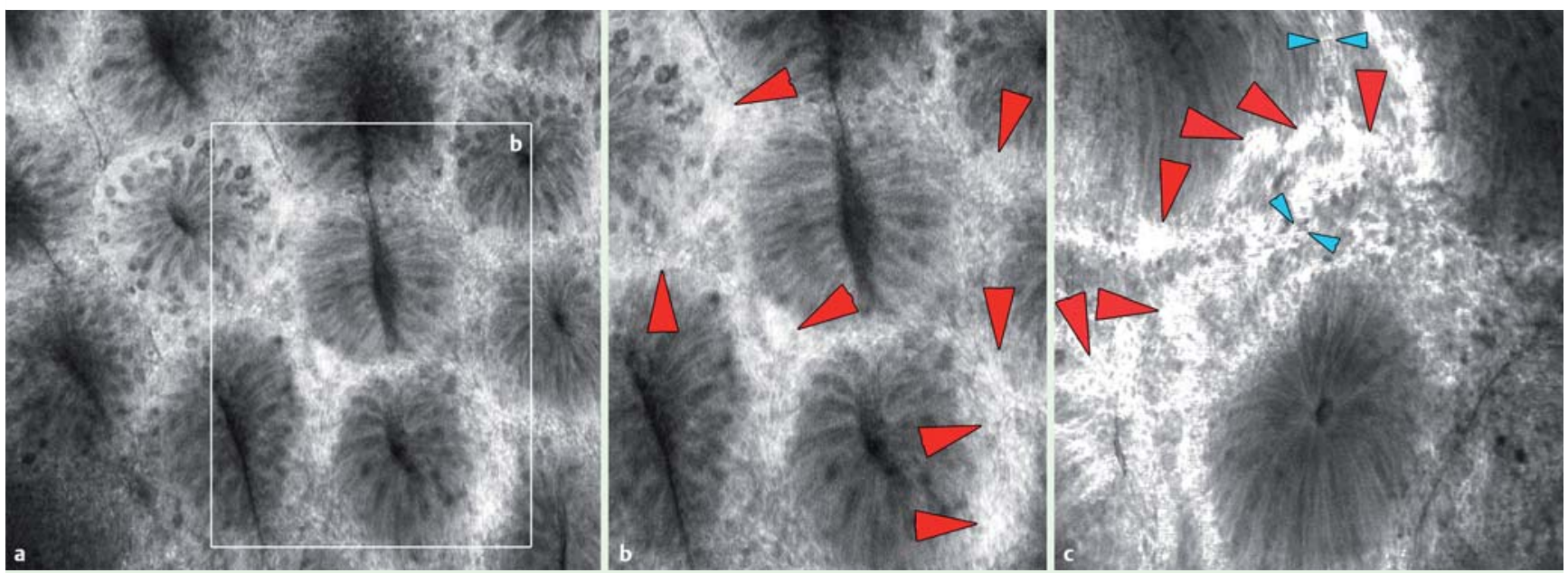

Fig.2 a Fluorescein-aided confocal endomicroscopy reveals an altered crypt architecture (asymmetrical crypts with elongated crypt lumen). b Higher magnification of the lamina propria between the crypts shows an inflammatory infiltrate (red arrowheads). c Confocal endopathology showing mild fluorescein leakage (red arrowheads) in the lamina propria as a sign of fluorescein extravasation from the vessels (between the blue arrowheads) in an inflamed area. 

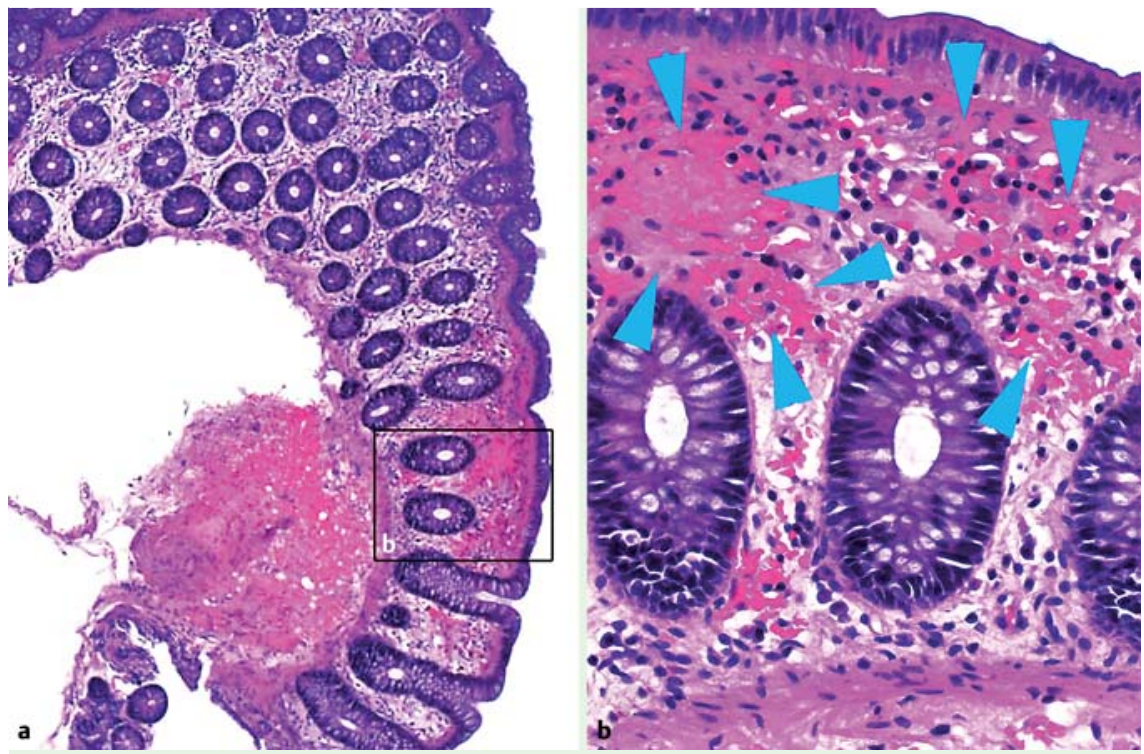

Fig. 3 a Overview of the colon biopsy (taken from the same area investigated by endomicroscopy) showing regenerative crypts with mild distorted and elongated lumina (hematoxylin eosin staining). b Prominent hemorrhagic edema (blue arrowheads) seen at higher magnification in the lamina propria, as well as numerous mononuclear cells and elongated crypts, confirming the endomicroscopic findings.
4 Beck KE, Blansfield JA, Tran KQ et al. Enterocolitis in patients with cancer after antibody blockade of cytotoxic T-lymphocyte-associated antigen 4. J Clin Oncol 2006; 24: $2283-2289$

5 Neumann H, Kiesslich R, Wallace $M B$ et al. Confocal laser endomicroscopy: technical advances and clinical applications. Gastroenterology 2010; 139: 388-392, 92 e1-2

Bibliography

DOI http://dx.doi.org/

10.1055/s-0031-1291603

Endoscopy 2012; 44: E78-E79

(c) Georg Thieme Verlag KG

Stuttgart · New York

ISSN 0013-726X

Corresponding author

\section{G. Hundorfean}

Medical Clinic I

University of Erlangen-Nuremberg

Ulmenweg 18

91054 Erlangen

Germany

Fax: +49-9131-8535102

gheorghe.hundorfean@uk-erlangen.de

\section{G. Hundorfean ${ }^{1}$, R. Atreya ${ }^{1}$, A. Agaimy², L. Heinzerling², E. Kämpgen ${ }^{3}$, G. Schuler ${ }^{3}$, M. F. Neurath ${ }^{1}$}

1 Department of Medicine I, University of Erlangen-Nuremberg, Erlangen, Germany

${ }^{2}$ Institute of Pathology, University of Erlangen-Nuremberg, Erlangen, Germany

${ }^{3}$ Department of Dermatology, University of Erlangen-Nuremberg, Erlangen, Germany

\section{References}

1 Sondak VK, Smalley KS, Kudchadkar $R$ et al. Ipilimumab. Nat Rev Drug Discov 2011; 10 : $411-412$

2 Salama AK, Hodi FS. Cytotoxic T-Lymphocyte-Associated Antigen-4. Clin Cancer Res 2011; 17: $4622-4628$

3 Hodi FS, O'Day SJ, McDermott DF et al. Improved survival with ipilimumab in patients with metastatic melanoma. $\mathrm{N}$ Engl J Med 2010; 363: $711-723$ 